\title{
Quantum Measures and States on Jordan Algebras
}

\author{
L. J. Bunce and J. D. Maitland Wright \\ Department of Mathematics, Reading University, Whiteknights Park, Reading, England
}

\begin{abstract}
A problem of Mackey for von Neumann algebras has been settled by the conjunction of the early work of Gleason and the recent advances of Christensen and Yeadon. We show that Mackey's conjecture holds in much greater generality. Let $A$ be a JBW-algebra and let $L$ be the lattice of all projections in $A$. A quantum measure on $L$ is a countably additive map, $m$, from $L$ into the real numbers. Our results imply that $m$ always has a unique extension to a bounded linear functional on $A$, provided that $A$ has no Type $I_{2}$ direct summand.
\end{abstract}

\section{Introduction}

Let $W$ be a von Neumann algebra or a JBW-algebra (see below for definitions). Let $P(W)$ be the lattice of all projections in $W$. A measure on $P(W)$ is a positive, realvalued function, $\mu$, on $P(W)$ such that $\mu(0)=0$ and, whenever $p$ and $q$ are orthogonal projections, $\mu(p+q)=\mu(p)+\mu(q)$. If, whenever $\left(p_{i}\right)$ is a countable family of orthogonal projections in $W, \mu\left(\sum p_{i}\right)=\sum \mu\left(p_{i}\right)$, then $\mu$ is said to be countably additive. Clearly, each positive linear functional on $W$ restricts to a measure on $P(W)$. When $W$ is the algebra of complex two-by-two matrices, or a spin factor, there exist measures on $P(W)$ which do not correspond to linear functionals on $W$.

Over twenty-five years ago, Mackey conjectured:

When $W$ is a von Neumann algebra with no Type $I_{2}$ direct summand and $\mu$ is any countably additive probability measure on $P(W)$ then $\mu$ can be extended to a state of $W$.

Very recently the problem of establishing Mackey's conjecture has been completely solved by Christensen [7] and Yeadon [33, 34]. Christensen used great ingenuity and insight to solve the problem for properly infinite von Neumann and for von Neumann algebras of Type $I_{n}$, where $3 \leqq n<\infty$. Yeadon devised different methods to deal with general finite von Neumann algebras and so complete the solution. We are grateful to the referee for drawing our attention to the work of 
Matveichuk who, independently, considered the problem for semifinite algebras [20]. The first major result had been obtained by Gleason [12] who solved the problem for Type $I$ factors. Important contributions were made by Gunson [14] who established crucial continuity properties of countably additive measures on continuous von Neumann algebras, and by Aarnes who worked with general $C^{*}$-algebras.

In most mathematical formulations of the foundations of quantum mechanics, the bounded observables of a physical system are identified with a real linear space, $L$, of bounded self-adjoint operators on a Hilbert space, $H$. Those bounded observables which correspond to the projections in $L$ form a complete orthomodular lattice, $P$, otherwise known as the lattice of "questions" or the quantum logic of the physical system. by

For $x, y$ in $B(H)_{s a}$, the physicist P. Jordan defined the Jordan product of $x$ and $y$

$$
x \circ y=\frac{1}{2}(x y+y x)=(x+y)^{2}-x^{2}-y^{2} .
$$

So it is reasonable to assume that $L$ is a Jordan algebra of self-adjoint operators on $H$ which is closed in the weak operator topology. Hence $L$ is a JBW-algebra. Mackey's Axiom VII, [19], makes the much stronger assumption that $L=B(H)_{s a}$. Mackey states that, unlike his other axioms, Axiom VII has no physical basis but is made for technical convenience. He goes to say "It would be interesting to have a thorough study of the consequences of modifying Axiom VII ... . One of the technical advantages of Axiom VII is that it allows us to use Gleason's theorem $[12]$ to identify the completely additive probability measures on $P$ with the normal states.

The main purposes of this paper is to extend the Gleason-Christensen-Yeadon theorem from von Neumann algebras to JBW-algebras. This removes one of the mathematical difficulties arising from weakening Axiom VII to the physically plausible assumption that $L$ is a JBW-algebra.

The main results of this paper may be summarized as follows:

Theorem. Let $M$ be a JBW-algebra with $P(M)$ its lattice of projections. Let $M$ be either Type $I_{n}$ for $3 \leqq n<\infty$, or Type $I_{\infty}$, or without Type I direct summand. Then any measure on $P(M)$ is the restriction of a positive linear functional on $M$.

Corollary. Let $M$ be a JBW-algebra with no Type $I_{2}$ direct summand. Then any countably additive measure on $P(M)$ is the restriction of a positive linear functional on $M$.

We make essential use of the methods of Christensen, Yeadon, Gunson, and Aarnes. It turns out that for the Type $I I_{1}$ case the methods of Yeadon can be generalized fairly easily. On the other hand, we need to surmount a number of technical obstacles before we can cope with the properly infinite JBW-algebras. Indeed, Type $I_{n}$, for $n \geqq 3$ gives rise to some non-trivial difficulties.

\section{Preliminaries}

A real Jordan algebra which is also a Banach space with a norm which satisfies $\left\|a^{2}-b^{2}\right\| \leqq \max \left(\left\|a^{2}\right\|,\left\|b^{2}\right\|\right),\left\|a^{2}\right\|=\|a\|^{2}$, for each pair of elements $a, b$ is said to be 
a JB-algebra. A JBW-algebra is a JB-algebra which is also the dual of a Banach space.

A uniformly closed Jordan algebra of self-adjoint operators on a Hilbert space is said to be a JC-algebra and is an example of a JB-algebra. A JW-algebra is a JCalgebra which is closed in the weak operator topology and is an example of a JBWalgebra. The classical example of a JB-algebra which lacks a Hilbert space representation is $M_{3}^{8}$, the $3 \times 3$ hermitian matrices over the Cayley numbers. Essentially, these account for all JB-algebras. In particular, each JBW-algebra, $M$, has a unique decomposition

$$
M=M_{\mathrm{ex}} \oplus M_{\mathrm{sp}},
$$

where $M_{\mathrm{ex}}$ is isomorphic to the real algebra $C_{\mathbb{R}}\left(X, M_{3}^{8}\right)$ of all $M_{3}^{8}$-valued continuous functions on some hyperstonean space $X$, and $M_{\mathrm{sp}}$ is (isomorphic to) a JW-algebra, [25, Theorem 3.9].

Each JW-algebra, $M$, has a unique decomposition of the form $I_{\text {fin }} \oplus I_{\infty} \oplus I I_{1} \oplus I I_{\infty} \oplus I I I, M$ being called properly infinite if its finite part $I_{\text {fin }} \oplus I I_{1}$ vanishes, $[2,31]$.

The Jordan triple product $\{a, b, c\}$ of elements $a, b, c$ in a Jordan algebra, $A$, is defined by $\{a, b, c\}=(a \circ b) \circ c+a \circ(b \circ c)-(a \circ c) \circ b$. The operators $U_{a}, L_{a}$ on $A$ are defined by $U_{a}(b)=\{a, b, a\}, L_{a}(b)=a \circ b$. One has $U_{a}=2 L_{a}^{2}-L_{a^{2}}$. If $A$ is a special Jordan algebra, these reduce to

$$
\{a, b, c\}=\frac{1}{2}(a b c+c b a), \quad\{a, b, a\}=a b a, \quad a \circ b=\frac{1}{2}(a b+b a) .
$$

The elements $a, b$ in $A$ are said to operator commute if $L_{a} L_{b}=L_{b} L_{a}$ on $A$. An element which operator commutes with all other elements is said to be central, and the set, in $A$, of all such elements is said to be the centre of $A$. Frequent (and tacit) use will be made of the well-known Jordan identities,

$$
\begin{gathered}
U_{\mathrm{U}_{a}(b)}=U_{a} U_{b} U_{a}, \quad\left(U_{a}(b)\right)^{2}=U_{a} U_{b}\left(a^{2}\right), \quad 4(a \circ b)^{2}=2 a \circ U_{b}(a) \\
+U_{a}\left(b^{2}\right)+U_{b}\left(a^{2}\right) .
\end{gathered}
$$

Further details of the theory of JB-algebras used here can be found in $[3-6,8,9$, $16,25,32]$. The reader is referred to $[2,10,26-29,31]$ for the relevant background on JC-algebras and to [15] for the general theory of Jordan algebras.

Given a JBW-algebra, $M$, and a subset, $S$, of $M$ we denote by $C[S]$ and $W[S]$, respectively, the JB-algebra and the JBW-algebra generated by $S$ in $M$. We write $C[S]=C\left[a_{2}, \ldots, a_{n}\right], W[S]=W\left[a, \ldots, a_{n}\right]$ if $S=\left\{a, \ldots, a_{n}\right\}$ is a finite set. The algebra $W[1, a, b]$, with $a, b$ in $M$, can be realized as a JW-algebra, as may be deduced from [32, Corollary 2;10];W[1,a] is the self-adjoint part of a commutative $W^{*}$-algebra, as is every associative JBW-algebra.

Definitions. Let $M$ be a JBW-algebra and let $P(M)$ be its projection lattice.

(a) A measure, $\mu$, on $P(M)$ will be said to be a measure on $M$ and a probability measure on $M$ if $\mu(1)=1$.

(b) A positive quasi-linear functional on $M$ is a mapping $\psi: M \rightarrow \mathbb{R}$ such that $\psi$ restricts to a positive linear functional on $C[x]$, for every $x$ in $M$. Also, $\psi$ is said to be a quasi-state if $\psi(1)=1$. 
The set of all positive quasi-linear functionals on, $\phi, M$, for which $\phi(1) \leqq 1$, is denoted by $Q(M)$. A quasi-state, $\psi$, is said to be pure if whenever $\alpha \in Q(M)$ with $\alpha \leqq \psi$, then $\alpha=\lambda \psi$ for some $\lambda \in[0,1]$. Since the functional calculus for JBWalgebras is the same as that for $W^{*}$-algebras, it is easily deduced, from arguments used in [1], that linear.

(i) If $M$ is associative then every positive quasi-linear functional on $M$ is

(ii) $Q(M)$ is a convex and compact set in the topology of pointwise convergence. The pure quasi-states are precisely the non-zero extreme points of $Q(M)$.

(iii) Each probability measure, $\phi$, on $M$ extends uniquely and canonically to a quasi-state, $\psi$, on $M$. The extension is expressed in the bijective correspondence given by

$$
\psi(a)=\int_{\sigma(a)} \lambda d \phi\left(e_{\lambda}\right), \quad \phi=\left.\psi\right|_{P(M)},
$$

where $\sigma(a)$ is the spectrum, and $\left(e_{\lambda}\right)$ is the spectral resolution, of the element $a$ of $M$.

\section{JBW-Factors of Type $I_{n}$, where $3 \leqq n<\infty$}

The first lemma is a technicality, the content of which occurs in various guises throughout the paper. Use will be made of the following identities. It can be varified that they hold in all special Jordan algebras. By Macdonald's theorem, $[15$, p. 40$]$, it follows that they are valid in every Jordan algebra.

$$
\begin{aligned}
& 4(x \circ y) \circ(x \circ z)=z \circ U_{x}(y)+y \circ U_{x}(z)+U_{x}(y \circ z)+\left\{z, x^{2}, y\right\}, \\
& 4 x \circ(y \circ(x \circ z))=z \circ U_{x}(y)+y \circ U_{x}(z)+U_{x}(y \circ z)+\left\{x^{2}, z, y\right\} .
\end{aligned}
$$

Recall that two projections $e, f$ of a JBW-algebra $M$ are said to be (Jordan) equivalent, written $e \sim f$, if there exist symmetries $s_{1}, \ldots, s_{n}$ in $M$ such that $U_{s_{1}} \ldots U_{s_{n}}(e)=f$. The projections $e, f$ are said to be exchanged by a symmetry $s$ if $U_{s}(e)=f$.

Lemma 2.1. Let $M$ be a $J B W$-algebra containing distinct projections $f, g$, e and a symmetry s such that $f \circ g \neq 0, e \circ f=e \circ g=0, U_{s}(f)=e, U_{f}(g) \in \mathbb{R} f, U_{g}(f) \in \mathbb{R} g$. Then $A=W[f, g, f \circ s] \cong M_{3}(\mathbb{R})_{s a}$.

Proof. If we knew already that $A$ was special, this would be quite straightforward. The general case seems to require a little more care. By appealing to the special Jordan algebras $W[f, s], W[e, s], W[g, s]$, where necessary, we obtain $U_{f}(s)$ $=U_{e}(s)=0 ; 2(s \circ f) \circ f=s \circ f=s \circ e=2(s \circ e) \circ e ; 4(s \circ f)^{2}=e+f, 4 U_{s \circ f}(f)=e$. In addition, $\quad 0=g \circ U_{e}(s)=U_{e}(g \circ s)=U_{s} U_{f}(g \circ s)$. So $U_{f}(g \circ s)=0$. Similarly, $U_{g}(f \circ s)=0$. We claim that $A=\operatorname{lin}\{f, g, e, f \circ g, f \circ s, g \circ(f \circ s)\}=B$, say. Indeed, the elements $f \circ(f \circ g)=\frac{1}{2}\left(U_{f}(g)+f \circ g\right), \quad g \circ(f \circ g)=\frac{1}{2}\left(U_{f}(g)+g \circ f\right), \quad(f \circ g)^{2}$ $=\frac{1}{2} f \circ U_{g}(f)+\frac{1}{4}\left(U_{f}(g)+U_{g}(f)\right), e \circ(f \circ s)=\frac{1}{2} f \circ s, e \circ\left(g \circ(f \circ s)=\frac{1}{2} g \circ(f \circ s)\right.$ all lie in $B$. Moreover, since $s$ operator commutes with both $f \circ s$ and $g \circ s$, we see that, from $g \circ U_{s}(f)=0, \quad 2 s \circ(s \circ f)=f+e, \quad(g \circ f) \circ s=2(g \circ(s \circ(s \circ f))) \circ s=2(g \circ s) \circ(s \circ(s \circ f))$ $=(g \circ s) \circ f+g \circ(s \circ f)$. Hence, $\{g, s, f\}=0$, and $\{s, f, g\}=2 g \circ(f \circ s)$. 
From (I1), (I2), respectively, we therefore deduce that $4(f \circ g) \circ(f \circ s)=s \circ U_{f}(g)$ $+2 g \circ(f \circ s), 4 f \circ(g \circ(f \circ s))=s \circ U_{f}(g)$, which belong to $B$. So, $g \circ(f \circ(g \circ(f \circ s)))$ $\in \mathbb{R} \cdot g \circ(f \circ s)$. Note also that, since $f \circ s$, $s$ operator commute,

$$
4 U_{s}\left(U_{s \circ f}(g)\right)=4 U_{s \circ(s \circ f)}(g)=U_{2 s \circ(s \circ f)}(g)=U_{e+f}(g)=U_{f}(g) .
$$

This implies that $4 U_{f \circ s}(g)=U_{s} U_{f}(g) \in \mathbb{R} e$. Therefore,

$$
(f \circ s) \circ(g \circ(f \circ s))=\frac{1}{2}\left(U_{f \circ s}(g)+(f \circ s)^{2} \circ g\right) \in \mathbb{R} e+\mathbb{R} f \circ g
$$

and, finally,

$$
4(g \circ(f \circ s))^{2}=2(f \circ s) \circ U_{g}(f \circ s)+U_{g}\left((f \circ s)^{2}\right)+U_{f \circ s}(g) \in \mathbb{R} g+\mathbb{R} e .
$$

It follows that $A$ is special, of dimension 6 and, since it must contain a factor of Type $I_{3}$, is isomorphic to $M_{3}(\mathbb{R})_{s a}$. The proof is complete. We remark that if, in Lemma $2.1, f=g$ or $f \circ g=0$, then $A \cong M_{2}(\mathbb{R})_{s a}$ or $\mathbb{R}+M_{2}(\mathbb{R})_{s a}$, respectively.

The Type $I_{2} \mathrm{JBW}$-factors are precisely the spin factors. There exists a spin factor with orthonormal dimension of any given cardinality. For $3 \leqq n<\infty$, each Type $I_{n} \mathrm{JBW}$-factor is isomorphic to $M_{3}^{8}$ or $M_{n}(\mathbb{F})_{s a}$, where $\mathbb{F}=\mathbb{R}, \mathbb{C}$ or the quaternion algebra, $\mathbb{H}$.

Theorem 2.2. Let $\phi$ be a measure on the Type $I_{n} J B W$-factor, $M$, where $3 \leqq n<\infty$. Then $\phi$ extends to a positive linear functional on $M$.

Proof. Consider the extension of $\phi$ to a positive quasi-linear functional on $M$, also denoted by $\phi$. In view of Gleason's theorem and the preceeding remarks, we may suppose that $M=M_{3}^{8}$ or $M_{n}(\mathbb{H})_{s a}$, where $\mathbb{H}$ is the quaternion algebra. We will deal with both of these types simultaneously.

First, for minimal projections $f, g$ in $M$, we will show that $\phi$ is linear on $W[f, g]$. We may suppose that $f \circ g \neq 0$. Since, by [3, Lemma 3.9], $f \vee g \neq 1$, we can find a minimal projection, $e$, in $M$ such that $e \leqq 1-f \vee g$. Further, according to [5, Lemma 6.3] there is a symmetry, $s$ in $M$ such that $U_{s}(f)=e$. Now Lemma 2.1 implies that $W[f, g] \subseteq W[f, g, f \circ s] \simeq M_{3}(\mathbb{R})_{s a}$. Therefore, by Gleason's theorem, $\phi$ is linear on $W[f, g]$.

Now let $p, x$ lie in $M$, where $p$ is a minimal projection of $M$. We will proceed to show that $\phi$ must be linear on $W[p, x]$.

The special Jordan algebra $W[x, p]$ has a centrally orthogonal decomposition into JW-factors,

$$
W[x, p]=A_{1} \oplus \ldots \oplus A_{r}, \quad \text { say } .
$$

Accordingly, $x=\sum_{e=1}^{r} x_{i}$, for some $x_{i} \in A_{i}$. Since $p$ is minimal, it must lie inside one of the $A_{i}$ : and we may suppose that $p \in A_{1}$. This means that the factor $A_{1}=W\left[x_{1}, p\right]$, and that for each $i \geqq 2$, the factors $A_{i}=W\left[x_{i}\right]$ are associative (and hence are isomorphic to $\mathbb{R}$ or $\{0\}$ ). Thus we need only show that $\phi$ is linear on the JW-factor $A_{1}$, which we may suppose to be non-associative.

Let, then, $A_{1}$ be a Type $I_{k} \mathrm{JW}$-factor, where $k \geqq 2$. Spectral theory demands that $x_{1}$ satisfy a polynomial of degree $\leqq k$. Since $U_{p}(M)=\mathbb{R} p$, and by using to advantage the fact that $A_{1}=W\left[x_{1}, p\right]$ is special, one can easily check that $A_{1}$ is 
linearly generated by the elements $p, x_{1}^{m}, p \circ x_{1}^{l},\left\{x_{1}^{r}, p, x_{1}^{s}\right\}$, where $m, l, r$, $s=1, \ldots, k-1$. Upon counting, we see that $A_{1}$ has dimension less than or equal to

$$
1+3(k-1)+\frac{1}{2}\left((k-1)^{2}-(k-1)\right)=\frac{1}{2} k^{2}+\frac{3}{2} k-1<2 k^{2}-k=\operatorname{dim}\left(M_{k}(H)_{s a}\right) .
$$

Thus $A_{1}$ cannot be the quaternionic Type $I_{k}$ factor. If $A_{1}=M_{k}(\mathbb{F})_{s a}, \mathbb{F}=\mathbb{R}$ or $\mathbb{C}$, for $k \geqq 3$, then our object is attained on applying Gleason's theorem. Thus it remains to consider the case when $A_{1}$ is a Type $I_{2}$ factor.

Suppose then that $A_{1}=W\left[x_{1}, p\right]$ is a spin factor, and let $h$ be the unit of $A_{1}$. This implies that $x_{1}=\alpha h+\beta q$ for some non-trivial projection, $q$, of $A_{1}$, where $\alpha, \beta$ are real numbers. Since $A_{1}$ is a spin factor, we have $h=p \vee q \in W[p, q] \subseteq A_{1}$, and so $W[p, q]=A_{1}$; and, moreover, $p$ and $q$ must be exchanged by a symmetry of $A_{1}$ (and hence by a symmetry of $M$, [31, Proposition 6]). Consequently, $q$, as well as $p$, must be a minimal projection of the whole algebra, $M$. Hence, by the first part of the proof, $\phi$ must be linear on $A_{1}=W[p, q]$; and so, by the above remarks, $\phi$ is linear on $W[x, p]$.

Finally, given any pair of elements $x, y$ of $M$ it now follows immediately, by using spectral theory and then iterating, that $\phi(x+y)=\phi(x)+\phi(y)$. This completes the proof.

\section{JBW-Algebras of Type $\boldsymbol{I}_{\boldsymbol{n}}, \mathbf{3} \leqq n<\infty$}

We begin with a brief survey of the properties of pure quasi-states and their connection with the problem in question. Brevity is appropriate because of the close similarity with the work of Aarnes [1].

Proposition 3.1. Each pure quasi-state on a JBW-algebra restricts to a pure state on the centre.

Proof. Let $M$ be a JBW-algebra. Then $Z(M)=C_{\mathbb{R}}(X)$, for some hyperstonean space $X$. Consequently, the argument from line 8 in [1, Sect. 5, Lemma 2, p. 614], with obvious modifications, implies that for any given pure quasi-state, $\phi$, of $M$ there exist $x_{0}$ in $X$ such that $\phi(h)=h\left(x_{0}\right)$ for every $h$ in $C(X)$. This means that $\phi$ is pure on the centre.

The primitive quotients of a JBW-algebra, $M$, of Type $I_{n}, 3 \leqq n<\infty$, are JBWfactors of the same type. By [30, Theorem 21] and [24, Theorem 3.9], it follows that $Z(M) \cong C_{\mathbb{R}}(X)$, where $X=\operatorname{Prim}(M)$. Moreover, also taking into account $[16$, Satz 36], for $n \geqq 3$ there exist hyperstonean spaces $X_{1}, Y_{1}, Y_{2}, Y_{3}$ for which

$$
M \cong C_{\mathbb{R}}\left(X, M_{3}^{8}\right) \oplus C_{\mathbb{R}}\left(Y_{1}, M_{n}(\mathbb{R})_{s a}\right) \oplus C_{\mathbb{R}}\left(Y_{2}, M_{n}(\mathbb{C})_{s a}\right) \oplus C_{\mathbb{R}}\left(Y_{3}, M_{n}(\mathbb{H})_{s a}\right),
$$

the first summand vanishing when $n>3$. Thus, bearing in mind the presence of the identity element wherever appropriate, the analogues, for $M$, of most of [1, Sect. 6] are easily obtained. In particular, we pick out:

Lemma 3.2 (cf. [1, Sect. 6, Lemma 2, p. 620]). Let $M$ be a JBW-algebra of Type $I_{n}$, $3 \leqq n<\infty$, and let $\phi$ be a pure quasi-state of $M$. Suppose that $Z(M) \cong C(X)$ and that $x_{0}$ is the point of $X$ for which $\phi(h)=h\left(x_{0}\right)$ for every $h$ in $Z(M)$. Then if $x, y$ are in $M$ which agree on a neighbourhood $U$ of $x_{0}$, we have $\phi(x)=\phi(y)$. 
Now running through the analogues (for JBW-algebras of Type $I_{n}, 3 \leqq n<\infty$ ) of [1, Sect. 6, Lemmas 3 and 4, Theorem 5] we get down to that of [1, Sect. 6, Corollary 3] which, on taking into account Theorem 2.2 and the Krein-Milman theorem, may be stated thus:

Theorem 3.3. Let $M$ be a JBW-algebra of Type $I_{n}, 3 \leqq n<\infty$. If all pure quasi-states on $M$ are continuous then all quasi-states on $M$ are linear.

If $M \leqq B(H)_{s a}$ is a JW-algebra, $R(M)$ and [M], respectively, will represent the real norm closed algebra and the $C^{*}$-algebra generated by $M$, with $R(M)^{-},[M]^{-}$ denoting the corresponding weak operator closures in $B(H)$. Recall that $M$ is said to be reversible if $a_{1} \ldots a_{n}+a_{n} \ldots a_{1}$ belongs to $M$ whenever $a_{1} \ldots a_{n}$ lie in $M$.

A projection $p$ is said to be faithful in the JBW-algebra $M$ if $c(p)=1$ [where $c(p)$ is the central support of $p$ in $M]$, it is said to be homogeneous of degree $n<\infty$ if $U_{p}(M)$ is Type $I_{n}$. Observe that if $p$ and $q$ are equivalent projections in $M$ then $c(p)$ $=c(q)$.

Definition. Projections $e, f$ in the JBW-algebra $M$ are isoclinic with angle $\alpha \in\left[0, \frac{\pi}{2}\right)$ if $U_{e}(f)=e \cdot \cos ^{2} \alpha, U_{f}(e)=f \cdot \cos ^{2} \alpha$.

Remarks 3.4. Let $e, f$ be projections in the JBW-algebra $M$. Then

(1) If $e, f$ are isoclinic, then $e, f$ are minimal in $W[e, f] \cong M_{2}(\mathbb{R})_{s a}$. Thus, there is $s$ in $W[e, f]$ with $s^{2}=e \vee f, U_{s}(e)=f, U_{s}(f)=e$, [5, Corollary 6.3]. Then $t=1+s-s^{2}$ is a symmetry in $M$ with $U_{t}(e)=f$.

(2) If $\|e-f\|<1$, then [31, Proposition 7] applied to the JW-algebra $W[1, e, f]$ implies that $U_{s}(e)=f$ for the symmetry $s=c^{-1 / 2} \circ(e+f-1)$, where $c=(e+f-1)^{2}$.

The results contained in the following lemma are proved for JW-algebras in [31, Theorem 10, Corollary 21, Lemma 26].

Lemma 3.5. Let $M$ be a JBW-algebra and let e, $f$ be projections in $M$. Then:

(i) There is a central projection $z$ and a symmetry $s$ in $M$ such that $U_{s}(e \circ z)$ $\leqq f \circ z, U_{s}(f \circ(1-z)) \leqq e \circ(1-z)$.

(ii) If $M$ has no infinite part and $e \sim f$, then e, $f$ are exchanged by a symmetry, in $M$.

(iii) If e, $f$ are abelian and $c(e)=c(f)$, then $e, f$ are exchanged by a symmetry.

Proof. (i) This can be obtained by straightforward modifications to [31, Corollary 16, Proposition 11, Lemmas 18 and 19, Theorem 19] together with inspection of the arguments in $[5$, Sect. 6].

(ii) By [25, Theorem 3.9] and [31, Corollary 21], $M$ can be supposed to be purely exceptional. Let $\pi$ be a factor representation of $M$ and let $s_{1}, \ldots, s_{n}$ be symmetries in $M$ such that $U_{s_{1}} \ldots U_{s_{n}}(e)=f$. Choose a central projection $c$ and a symmetry $s$ satisfying (i). Since $\pi M=M_{3}^{8}$, it follows that $\pi(f \circ z)=U_{\pi\left(s_{1}\right)} \ldots U_{\pi\left(s_{n}\right)} U_{\pi(s)}(\pi(f \circ z))$. Therefore, by [5, Corollary 5.7], $f \circ z=U_{s_{1}} \ldots U_{s_{n}} U_{s}(f \circ z)$. Hence $e \circ z=U_{s}(f \circ z)$. Similarly, $e \circ(1-z)=U_{s}(f \circ(1-z))$. So, $U_{s}(e)=f$.

(iii) Choose central projection $z$ and a symmetry $s$ satisfying (i). In particular, $p=U_{s}(e \circ z) \leqq f \circ z=q$. Both $p, q$ are abelian and $c(p)=c(q)$. By the proof of $[9$, Theorem, p. 322], $p=q c(p)$. Hence $p=q$. Similarly, $U_{s}(f(1-z))=e \circ(1-z)$. Hence $U_{s}(e)=f$. 
Lemma 3.6. Let $f, g$ be projections in the $J B W$-algebra, $M$, and suppose that $\|f-g\|<1$ and that there is a projection $e$ in $M$ such that $e \sim f, e \circ f=e \circ g=0$. Suppose also that the angle $\alpha \in[0, \pi / 4)$ and $\sin ^{-1}\left\{U_{f}(1-g)^{1 / 2}\right\} \leqq 2 \alpha f$. Then there exists a projection $h$ in $M$ isoclinic with angle $\alpha$ to both $f$ and $g$. In particular, this holds when $\alpha=\frac{1}{2} \sin ^{-1}\left\|U_{f}(1-g)^{1 / 2}\right\|$.

Proof. There exist central projections $e_{1}, e_{2}, e_{3}$ in $M$, where (up to isomorphism) $M_{\mathrm{rev}}=e_{1} \circ M$ is a reversible JW-algebra, $M_{2}=e_{2} \circ M$ is a Type $I_{2} \mathrm{JW}$-algebra and $M_{\mathrm{ex}}=e_{3} \circ M$ is purely exceptional. Since

$$
\sin ^{-1}\left\{U_{f \circ e_{i}}\left(1-g \circ e_{i}\right)^{1 / 2}\right\}=e_{i} \circ \sin ^{-1}\left\{U_{f}(1-g)^{1 / 2}\right\} \leqq 2 \alpha f \circ e_{i}, \quad i=1,2,3,
$$

it is enough to prove the result separately for each of the cases $M=M_{2}, M_{\mathrm{rev}}, M_{\mathrm{ex}}$.

(a) $M=M_{2}$. Let $\pi$ be a factor representation of $M$. Then $\pi(M)$ is a spin factor, [26, Lemma 2] since $\pi(e) \sim \pi(f) \sim \pi(g)$ and $\pi(e) \circ(\pi(f)+\pi(g))=0$ and since all projections $\neq 0,1$ in a spin factor are minimal, it follows that $\pi(f-g)=0$. Hence $f=g$, by [5, Corollary 5.7]. By [31, Corollary 21], or Lemma 3.4(ii), there is a symmetry, $s$, in $M$ such that $U_{s}(e)=f$. Put $h=f \cdot \cos ^{2} \alpha+2\{f, s, e\} \sin \alpha \cos \alpha$ $+e \sin ^{2} \alpha$. Calculation shows that $h$ is a projection and that $U_{h}(f)=h \cdot \cos ^{2} \alpha, U_{f}(h)$ $=f \cdot \cos ^{2} \alpha$.

(b) $M=M_{\mathrm{rev}}$. Inspection of the arguments in [14, Lemma 2.6] and [7, Lemma 2.3] reveals that the discussions there can be conducted entirely in $R(M)^{-}$. The facts that one needs to observe are that the elements $W, W^{\prime}$ in [14, Lemma 2.6], can be chosen in $R(M)^{-}$. Indeed, $W|x|$, is the polar decomposition in $[M]^{-}$of $x=(1-e) f e \in R(M)$. Consequently (see, for example, the proof [21, 2.2.9]), $W$ is the strong limit of the sequence $x(1 / n+|x|)^{-1}$, lying in $R(M)$. Hence $W \in R(M)^{-}$. In addition, because there exist symmetries $s_{1}, \ldots, s_{n}$ in $M$ such that $s_{1} \ldots s_{n} e s_{n} \ldots s_{1}=f$, we can choose $W^{\prime}=f s_{n} \ldots s_{1} \in R(M)$. It then follows that the relevant projection $h \in R(M)_{s a}^{-}=M$, because $M$ is reversible [27, Remark 2.5].

(c) $M=M_{\mathrm{ex}}$. By Lemma 3.5(ii), there is a symmetry $s$ in $M$ with $U_{s}(e)=f$. Consider the JBW-subalgebra of $M, A=W[f, g, f \circ s]$. Using the fact that $W[f, s], W[e, s]$ are JW-algebras, one obtains $U_{f}(s)=U_{e}(s)=0 ; 2(s \circ f) \circ f=s \circ f$ $=s \circ e=2(s \circ e) \circ e ;(2(s \circ f))^{2}=e+f, U_{2(s \circ f)}(f)=e$. Therefore, $e$ and $f$ are equivalent in $A$. In fact, $U_{t}(e)=f, t^{2}=1_{A}$, where $t=1_{A}+2 f \circ s-e-f\left(1_{A}\right.$ being the identity element of $A$ ). Thus in view of (a), (b) and the remarks preceding them, the proof will be complete once it is shown that $A$ is (isomorphic to) a JW-algebra.

First, observe that $e, f$, and $g$ are abelian projections in $M$, and consequently in $A$. Indeed, for any factor representation $\pi$ of $M, \pi(M)=M_{3}^{8}$ and so either $\pi(f), \pi(e)$, $\pi(g)$ are either all minimal or all zero. Now use [6, Lemma 3.2].

Consider now the JB-subalgebra of $A, B=C\left[1_{A}, f, g, f \circ t\right]$, and let $\psi: B \rightarrow N$ be any factor representation. $B$ contains $e, t$, and either $\psi(e), \psi(f), \psi(g)$ are all minimal or all zero. Therefore, $N \cong \mathbb{R}$ or $N=W[\psi(f), \psi(g), \psi(f) \circ \psi(t)]$ and the latter has dimension $\leqq 6$, by Lemma 2.1 and the remarks following it. Hence $B$ is a JC-algebra, by [5, Theorem 9.5]. Consequently, $A$, which is the $\sigma\left(M, M_{*}\right)$ closure of $B$ (where $M_{*}$ is the predual of $M$ ), is a JW-algebra, being a quotient of the JWalgebra $B^{* *}$.

The proof of the final statement in the lemma is contained in [7, Lemma 2.3]. This completes the proof. 
Corollary 3.7. Let $f, g$ be projections in a JBW-algebra $M$ of Type $I_{n}$, where $3 \leqq n<\infty$. Suppose that $\|f-g\|<\frac{1}{2}$ and that $f$ is faithful and homogeneous of degree $k$ in $M$. Then for any measure $\phi$ on $M,|\phi(f)-\phi(g)| \leqq 2 \sqrt{2} \cdot k \cdot\|f-g\|^{1 / 2}$.

Proof. The symmetry $s=c^{-1 / 2} \circ(f+g-1)$, where $c=(f+g-1)^{2}$, exchanges $f$ and $g$, by Remarks 3.4(2). Operating in the JW-algebra $W[1, f, g]$ one calculates that $U_{f}(s)=c^{-1 / 2} \circ a=a^{1 / 2}$, where $a=U_{f}(g)$. By assumption, $f=p_{1}+\ldots+p_{k}$, for some faithful abelian projections $p_{1}, \ldots, p_{k}$. Put $q_{i}=U_{s}\left(p_{i}\right)$. Then the $q_{i}$ are faithful abelian and $q=q_{1}+\ldots+q_{k}$. Observe that

$$
\left\|p_{i}-q_{i}\right\|^{2}=\left\|U_{p_{i}}\left(1-q_{i}\right)+U_{1-p_{i}}\left(q_{i}\right)\right\|=\max \left(\left\|U_{p_{i}}\left(1-q_{i}\right)\right\|,\left\|U_{q_{i}}\left(1-p_{i}\right)\right\|\right),
$$

and that

$$
\left\|p_{i}-U_{p_{i}}\left(a^{1 / 2}\right)\right\|=\left\|U_{p_{i}}\left(f-a^{1 / 2}\right)\right\| \leqq\left\|f-a^{1 / 2}\right\| \leqq\|f-a\| \leqq\|f-g\|
$$

So,

$$
\left\|U_{p_{i}}\left(1-q_{i}\right)\right\|=\left\|p_{i}-U_{p_{i}} U_{f} U_{s} U_{f}\left(p_{i}\right)\right\|=\left\|p_{i}-\left(U_{p_{i}}\left(a^{1 / 2}\right)\right)^{2}\right\| \leqq 2\|f-g\| .
$$

Therefore, $\left\|p_{i}-q_{i}\right\| \leqq \sqrt{2} \cdot\|f-g\|^{1 / 2}<1$, by symmetry.

Fix $i$. By Lemma 3.6, there exists a projection $h_{i}$ isoclinic to both $p_{i}$ and $q_{i}$ with angle $\alpha=\sin ^{-1}\left\|U_{p_{i}}\left(1-q_{i}\right)^{1 / 2}\right\|$. By Remarks 3.4(1), $h_{i}$ is also faithful and abelian in $M$. This means that there is a projection $e_{i} \leqq 1-p_{i} \vee h_{i}$, which is faithful abelian in $M$ and, consequently, a symmetry $s_{i}$ exchanging $e_{i}$ and $p_{i}$, by Lemma 3.5(iii). Thus, $W\left[p_{i}, h_{i}, p_{i}{ }^{\circ} s_{i}\right] \cong M_{3}(\mathbb{R})_{\text {sa }}$, by Lemma 2.1. Similarly, $q_{i}, h_{i}$ can be embedded in a copy of $M_{3}(\mathbb{R})_{s a}$ contained in $M$. Applying Gleason's theorem, as in the relevant part of $\left[7\right.$, Proposition 2.3], it follows that $\left|\phi\left(p_{i}\right)-\phi\left(q_{i}\right)\right| \leqq 2\left\|p_{i}-q_{i}\right\|$. Therefore,

$$
\|\phi(f)-\phi(g)\| \leqq \sum_{i=1}^{k}\left|\phi\left(p_{i}\right)-\phi\left(q_{i}\right)\right| \leqq 2 \cdot \sqrt{2} \cdot k \cdot\|f-g\|^{1 / 2},
$$

and the proof is complete.

Theorem 3.8. Let $M$ be a Type $I_{n} J B W$-algebra, where $3 \leqq n<\infty$. Then every measure on $M$ extends to a positive linear functional on $M$.

Proof. We are now in a position to adapt [7, Theorem 3.1] more or less directly. Indeed, suppose that $\phi$ is a pure quasi-state on $M$. By [16, Satz 36] and [25, Theorem 3.9], we may suppose that $M=C_{\mathbb{R}}\left(X, M_{n}(\mathbb{F})_{s a}\right)$, where $\mathbb{F}=\mathbb{R}, \mathbb{C}, \mathbb{H}$ or the Cayley numbers; and $X$ is some hyperstonean space. Then $\phi$ is concentrated at some point $x_{0}$ of $X$, in the sense of Lemma 3.2. Given $c, d$ in $M, \varepsilon>0,\|c-d\|<\varepsilon$, an hyperstonean neighbourhood, $V$, of $x_{0}$ can be chosen so that

$$
|\phi(c)-\phi(d)| \leqq \varepsilon / 2+\sum_{i=1}^{m} \lambda_{i}\left|\phi\left(p_{i}\right)-\phi\left(q_{i}\right)\right|, \quad \text { where } \quad m \leqq n
$$

for certain positive real numbers $\lambda_{i}$ and projections $p_{i}, q_{i}$ which are faithful and homogeneous, of degree $k_{i}=$ dimension $p_{i}\left(x_{0}\right)$, in $N=C\left(V, M_{n}(\mathbb{F})_{s a}\right)$ satisfying

$$
\left\|p_{i}-q_{i}\right\| \leqq \frac{\varepsilon^{2}}{2 n^{2}}\left(4 k_{i}\left(1+\sum_{i=1}^{m} \lambda_{i}\right)\right)^{-2}
$$


Thus $|\phi(c)-\phi(d)|<\varepsilon$. Therefore, the desired conclusion results from Lemma 3.7 and Theorem 3.3.

\section{Properly Infinite JW-Algebras}

The reader is referred to [2] and [31] for an account of the relevant properties of finite and infinite JW-algebras (called, respectively, modular and non-modular in [31]). In order to apply the techniques of Christensen, [7], we first need to establish certain technical properties of JW-algebras.

The JC-algebra $A$ is isomorphic to a generating JC-subalgebra of its universal enveloping $C^{*}$-algebra, $C^{*}(A),[4$, Theorem 5.2]. By abstract nonsense, if $A$ and $B$ are isomorphic $\mathrm{JC}$-algebras then $C^{*}(A)$ and $C^{*}(B)$ are ${ }^{*}$-isomorphic $C^{*}$-algebras.

Suppose that $A$ is a finite dimensional JC-algebra. Then $C^{*}(A)$ is finite dimensional, and we may make the identifications (see [13, p. 90] and [24, p. 355])

$$
C_{\mathbb{R}}(X, A)=C_{\mathbb{R}}(X) \otimes_{\mathbb{R}} A, \quad C_{\mathbb{C}}\left(X, C^{*}(A)\right)=C_{\mathbb{C}}(X) \otimes_{\mathbb{C}} C^{*}(A),
$$

where $X$ is a compact Hausdorff space. We may also regard $A$ and $C_{\mathbb{R}}(X) \otimes_{\mathbb{R}} A$ as being contained in and generating $C^{*}(A)$ and $C_{\mathbb{C}}(X) \otimes_{\mathbb{C}} C^{*}(A)$, respectively.

Let $B$ be a $C^{*}$-algebra and let $\pi: C_{\mathbb{R}}(X) \otimes_{\mathbb{R}} A \rightarrow B_{\text {sa }}$ be a Jordan homomorphism. By the universal property the induced Jordan homomorphisms

$$
\pi_{1}: C_{\mathbb{R}}(X) \rightarrow B_{s a}(x \rightarrow \pi(x \otimes 1)), \quad \pi_{2}: A \rightarrow B_{s a}(a \rightarrow \pi(1 \otimes a)),
$$

extend to *-homomorphisms $\bar{\pi}_{1}: C(X) \rightarrow B$ and $\bar{\pi}_{2}: C^{*}(A) \rightarrow B$, respectively. In turn these induce a linear mapping

$$
\bar{\pi}: C_{\mathbb{C}}(X) \otimes_{\mathbb{C}} C^{*}(A) \rightarrow\left(x \otimes a \rightarrow \bar{\pi}_{1}(x) \cdot \bar{\pi}_{2}(a)\right),
$$

which clearly extends $\pi$. From the fact that $A$ generates $C^{*}(A)$ it is easy to see that $\bar{\pi}$ is actually a *-homomorphism. It follows that $C^{*}\left(C_{\mathbb{R}}(X, A)\right)=C_{\mathbb{C}}\left(X, C^{*}(A)\right)$.

In addition, let us note that by [11, Corollary 5.1],

$$
C^{*}\left(C_{\mathbb{R}}(X, A)\right)^{* *} \cong C_{\mathbb{C}}\left(X, C^{*}(A)\right)^{* *} \cong C_{\mathbb{C}}(X)^{* *} \otimes_{\mathbb{C}} C^{*}(A)^{* *} \cong C(Y) \otimes_{\mathbb{C}} C^{*}(A)^{* *},
$$

for some hyperstonean space $Y$, whenever $A$ is finite dimensional.

Let $V_{n}$ denote a spin factor of orthonormal dimension $n+1$, where $2 \leqq n<\infty$. By $\left[15\right.$, pp. 75 and 263], $C^{*}\left(V_{2 n}\right)=M_{2^{n}}(\mathbb{C})$ and $C^{*}\left(V_{2 n+1}\right)=M_{2^{n}}(\mathbb{C}) \oplus M_{2^{n}}(\mathbb{C})$. Using [28, Corollary 6.5] and [29, Corollary 3.5], for example, it can be seen that $C^{*}\left(M_{n}(\mathbb{F})_{s a}\right)=M_{n}(\mathbb{C}), M_{n}(\mathbb{C}) \oplus M_{n}(\mathbb{C})$ or $M_{2 n}(\mathbb{C})$, respectively, according to whether $\mathbb{F}=\mathbb{R}, \mathbb{C}$ or $\mathbb{H}$, for $n \geqq 3$.

The following lemma is an immediate consequence of the above remarks.

Lemma 4.1. Let $A$ be a JC-algebra and $X$ a compact Hausdorff space. Then $[A]^{-}$is

(i) Type $I_{n}$ if $A \cong C\left(X, M_{n}(\mathbb{F})_{\text {sa }}\right)$, where $\mathbb{F}=\mathbb{R}$ or $\mathbb{C}$.

(ii) Type $I_{2 n}$ if $A \cong C\left(X, M_{n}(\mathbb{H})_{s a}\right)$.

(iii) Type $I_{2^{n}}$ if $A \cong C\left(X, V_{k}\right)$, where $k=2 n$ or $2 n+1$.

We will now prove a sequence of lemmas aimed at Theorem 4.6.

Lemma 4.2. Let $M \subseteq B(H)_{s a}$ be a JW-algebra and let $p, q$ be projections in $M$. Suppose that the identity of $B(H)$ is contained in $M$ and that $N$ is a $J W$-subalgebra of 
$M$ containing 1 and isomorphic to $M_{3}(\mathbb{R})_{\text {sa }}$ such that $N \cong\{p, q\}^{\prime} \cap M$. Then the $J W$ algebra $Q=W[\{p, q\} \cup N]$ has central summands of Type $I_{3}$ or Type $I_{6}$ only.

Proof. Using Lemma 4.1, $M_{3}(\mathbb{C}) \cong[N] \cong\{p, q\}^{\prime} \cap[M]^{-}$. Since $L=[N]$ commutes elementwise with $\{p, q\}^{\prime \prime}$, there is an algebraic surjective *-homomorphism from $\{p, q\}^{\prime \prime} \otimes L$ onto the algebra generated by $\{p, q\}^{\prime \prime}$ and $L$. In addition, from [30, Chap. V, Theorem 1.41] and the reasoning employed in the preamble to Lemma 4.1, there exist algebraic *-isomorphisms

$$
\begin{aligned}
\{p, q\}^{\prime \prime} \otimes L & \cong\left[C_{\mathbb{C}}(X) \oplus C_{\mathbb{C}}\left(Y, M_{2}(\mathbb{C})\right)\right] \otimes M_{3}(\mathbb{C}) \\
& \cong C_{\mathbb{C}}(X) \otimes M_{3}(\mathbb{C}) \oplus\left(C_{\mathbb{C}}(Y) \otimes M_{2}(\mathbb{C}) \otimes M_{3}(\mathbb{C})\right. \\
& \cong C_{\mathbb{C}}\left(X, M_{3}(\mathbb{C})\right) \oplus C_{\mathbb{C}}\left(Y, M_{6}(\mathbb{C})\right)=A, \text { say },
\end{aligned}
$$

for certain hyperstonean spaces $X, Y$. Therefore, since the algebra generated by $\{p, q\}$ and $L$ is weakly dense in $\{\{p, q\}\} \cup L\}^{\prime \prime}=[Q]^{-}$, there exist hyperstonean spaces $S, T$ such that $C_{\mathbb{C}}\left(S, M_{3}(\mathbb{C}) \oplus C_{\mathbb{C}}\left(T, M_{6}(\mathbb{C})\right) \cong A^{* *} \stackrel{\phi}{\longrightarrow}[Q]^{-}\right.$, where $\phi$ is a $w^{*}$-continuous surjective ${ }^{*}$-homomorphism. The desired conclusion is now an immediate consequence of [29, Theorem $6.4 ; 6$, Proposition $4.5 ; 16$, Satz 36] and Lemma 4.1.

Lemma 4.3. Let $M \cong B(H)_{s a}$ be a properly infinite $J W$-algebra, containing the identity element of $B(H)$ and such that $R(M)^{-} \cap i R(M)^{-}=0$. Suppose that $N \cong M_{3}(\mathbb{R})_{\text {sa }}$ is a $J W$-subalgebra of $M$ containing 1 . Then $M \cap N^{\prime}$ is a properly infinite $J W$-algebra.

Proof. $M$ is reversible, by [28, Theorems 6.4 and 6.6] and $[M]^{-}$is a properly infinite von Neumann algebra, by $\left[2\right.$, Theorem 8]. Since $1 \in[N] \cong M_{3}(\mathbb{C})$, $[M]^{-} \cap N^{\prime}=[M]^{-} \cap[N]^{\prime}$ is also properly infinite.

Consider the real algebra $R=R(M)^{-} \cap N^{\prime}$. Then $R \cap i R=0$. By [29, Theorem 2.4], $[M]^{-}=R(M)^{-} \oplus i R(M)^{-}$, and it follows that $R \oplus i R=[M]^{-} \cap N^{\prime}$. In addition, since $R(M)_{s a}^{-}=M$, we find that $R_{s a}=M \cap N^{\prime}$.

If $M \cap N^{\prime}$ is not properly infinite then it has a non-zero finite normal trace, $\phi$. But then, on applying [2, Theorem 1] to $R, \phi$ must extend to a finite normal trace on the properly infinite algebra $[M]^{-} \cap N^{\prime}$, a contradiction. Therefore, $M \cap N^{\prime}$ is properly infinite, completing the proof.

Suppose that $M$ is a properly infinite JW-algebra. Since $[M]^{-}$is properly infinite, there is a projection $e$ in $[M]^{-}$with $e \sim 1-e \sim 1$ (where here $\sim$ denotes the usual von Neumann equivalence). The intention of the following digression (it will be needed in Theorem 4.6) is to show how $e$ may be chosen in $M$ with a measure of control over the partial isometries implementing the equivalences. The usual Jordan equivalence is inadequate for this purpose.

Because, for technical reasons, we need consider no other kind, we will confine our attention to reversible JW-algebras and avoid unnecessary qualifications.

Let $M$ be a reversible JW-algebra and let $e, f$ be projections in $M$. We will write $e \approx f$ if there exists a partial isometry $u$ in $R(M)^{-}$such that $e=u^{*} u, f=u u^{*}$. If there exists $u$ in $R(M)^{-}$such that $e=u^{*} u, u u^{*} \leqq f$, we will write $e \leqq f$. [Note that $u u^{*} \in M$, because $R(M)_{s a}^{-}=M$.] 
It is easy to see that if in a reversible JW-algebra, $M, e, f$ are Jordan equivalent, then $e \approx f$ and, moreover, that $\approx$ is an equivalence relation amongst the projections of $M$.

Lemma 4.4. Let $M$ be a reversible $J W$-algebra. Then,

(i) If $\left(e_{i}\right)_{i \in I},\left(f_{i}\right)_{i \in I}$ are both families of mutually orthogonal projections in $M$ such that $e_{i} \approx f_{i}$, for every $i$ in $I$, then $\sum e_{i} \approx \sum f_{i}$.

(ii) If e,f are projections in $M$ such that $e \geqq f, f \lesssim e$, then $e \approx f$.

(iii) If e, $f$ are projections in $M$, then there exists a central projection $z$ in $M$ such that $e z \lesssim f z, f(1-z) \lesssim e(1-z)$.

Proof. (i) Let $u_{i} \in R(M)^{-}$such that $e_{i}=u_{i}^{*} u_{i}, f_{i}=u_{i} u_{i}^{*}, i \in I$. Then $\sum_{e \in I} u_{i}$ lies in $R(M)^{-}$and implements the equivalence $\sum_{e \in I} e_{i} \approx \sum_{i \in I} f_{i}$.

(ii) Let $u, v \in R(M)^{-}$such that $e=u^{*} u, u u^{*}=f_{1} \leqq f, f=v^{*} v, v v^{*}=e_{1} \leqq e$. Then, in view of (i), inspection of the argument in [30, Chap. V, Proposition 1.3] yields $e \approx f$.

(iii) By [31, Theorem 19] (see also Lemma 3.1), there exists a symmetry $s$ and a central projection $z$ in $M$ with $s e z s \leqq f z$, se $(1-z) s \leqq e(1-z)$. Put $u=s e z$, $v=s f(1-z)$. The result follows.

A projection $e$ is a $\mathrm{JBW}$-algebra $M$ is said to be properly infinite if $e M e$ is a properly infinite JW-algebra.

Proposition 4.5. Let e be a projection in a reversible JW-algebra $M$. Then the following are equivalent.

(i) $e$ is properly infinite in $M$.

(ii) There exists an infinite sequence $\left(p_{n}\right)$ of mutually orthogonal projections in $M$ such that $\sum_{n=1}^{\infty} p_{n}=e \approx p_{m}$, for every $m$.

(iii) There exists a projection $f$ in $M$ with $e \geqq f \approx e \approx e-f$.

Proof. (i) $\Rightarrow$ (ii): We may suppose that $e=1$ is properly infinite. By [31, Lemma 23], there exists an infinite sequence, $\left(r_{n}\right)$, of mutually orthogonal Jordan equivalent projections in $M$. In particular, $r_{n} \approx r_{m}$, for all $m, n$. From this fact and Lemma 4.4 it can be shown as in $[22,2.2 .4]$ with only formal notational changes, that there is, in $M$ a central projection, $z$, and in infinite sequence, $\left(p_{n}\right)$, of mutually orthogonal projections such that $\sum_{n=1}^{\infty} p_{n}=z \approx p_{m}$, for every $m$. Since $(1-z) \circ M$ is properly infinite, the result follows by transfinite induction.

(ii) $\Rightarrow$ (iii): This follows immediately from Lemma 4.4(ii).

(iii) $\Rightarrow$ (i): Assume (iii). Then $e[M]^{-} e$ is a properly infinite von Neumann algebra. In order to deduce (i) we may suppose that $R(M)^{-} \cap i R(M)^{-}=0$, by $[28$, Lemma 6.1]. Applying [2, Theorem 1] to the real algebra $R=e R(M)^{-} e$, it follows that if $e M e$ has a non-zero finite normal trace then so does $e[M]^{-} e$, a contradiction. Therefore, $e M e$ is properly infinite.

Mackey's conjecture for properly infinite JW-algebras can now be established by applying the methods of Christensen [7, Theorem 4.1]. For completeness we indicate the proof. 
Theorem 4.6. Every measure $\phi$ on a properly infinite $J W$-algebra $M$ extends to a positive linear functional on $M$.

Proof. We may suppose that $M \subseteq B(H)_{\text {sa }}$, where $M$ contains the unit of $B(H)$ and that $\phi$ is a quasi-state on $M$. The fact that $M$ is reversible (see above), so that $R(M)_{s a}=M$, will be used tacitly. Applying [7, Theorem 4.1] and [28, Lemma 6.1] together, we may further assume that $R(M)^{-} \cap i R(M)^{-}=0$.

Now suppose that $N$ is a JW-subalgebra of $M$ such that $1 \in N \cong M_{3}(\mathbb{R})_{\text {sa }}$, and consider the JW-algebra $Q=M \cap N^{\prime}$. Using Lemma 4.3 and Proposition 4.5, there can be chosen in $M$ an increasing sequence, $\left(p_{n}\right)$, of mutually orthogonal projections such that $p_{n} \approx 1 \approx 1-p_{n}($ in $Q), \phi\left(1-p_{n}\right) \leqq 2^{-n}$ for every $n$. Next, given $a, b$ in $M$ with $0 \leqq a, b \leqq \frac{1}{2} p_{n}$, and $\varepsilon>0$, choose $k>n$ such that $\phi\left(1-p_{k}\right)<(\varepsilon / 6)^{2}$. As in $\left[7\right.$, Theorem 4.1], we find orthogonal projections $q, r$ such that $a=p_{n} q p_{n}, b=p_{n} r p_{n}$, and by Proposition 4.5, we can ensure that $q, r$ lie in $Q$. By Theorem 3.8 and Lemma $4.2, \phi$ restricts to a positive linear functional on $W\left[p_{n}, q\right]$. Extend this to a positive linear functional $\psi$ on the $C^{*}$-algebra, $\left[W\left[p_{n}, q\right]\right]$. Then

$$
|\phi(q)-\phi(a)|=\left|\psi\left(\left(1-p_{n}\right) q p_{n}\right)+\psi\left(q\left(1-p_{n}\right)\right)\right| \leqq \varepsilon / 3 .
$$

Treating $q+r, r$ similarly, one gets $|\phi(a+b)-\phi(a)-\phi(b)|<\varepsilon$ and proceeds to show that $\phi$ is linear on $p_{n} Q p_{n}$ exactly as in [7, Theorem 4.1].

As above, there exists, in $M$, an increasing sequence of projections, $\left(e_{n}\right)$, such that $e_{n} \approx 1-e_{n} \approx 1$ and $\phi\left(e_{n}\right) \uparrow 1$. Choose orthogonal projections $f_{n}, g_{n} \leqq 1-e_{n}$, in $M$, such that $1-e_{n}=f_{n}+g_{n} \approx f_{n} \approx g_{n}$. Then there are partial isometries $u_{n}, v_{n}$ in $R(M)^{-}$ such that $u_{n}^{*} u_{n}=v_{n}^{*} v_{n}=e_{n}, u_{n} u_{n}^{*}=f_{n}, v_{n} v_{n}^{*}=g_{n}, e_{n}+u_{n} u_{n}^{*}+v_{n} v_{n}^{*}=1$. One can easily check that the real linear space, $\operatorname{lin}\left\{e_{n}, f_{n}, g_{n}, u_{n}+u_{n}^{*}, v_{n}+v_{n}^{*}, u_{n} v_{n}^{*}+v_{n}^{*} u_{n}\right\}=N_{n}$, contained in $M$, is a JW-algebra isomorphic to $M_{3}(R)_{s a}$. Since $\phi$ is linear on $M \cap N_{n}^{\prime}$, and since $e_{n} x e_{n}+u_{n} x u_{n}^{*}+v_{n} x v_{n}^{*} \in M \cap N_{n}^{\prime}$, for all $x$ in $M$, the proof can now be completed as in the final paragraph of [7, Theorem 4.1].

\section{Type $I I_{1}$ JW-Algebras}

Let $M$ be a Type $I I_{1} \mathrm{JW}$-algebra. Then $M$ is reversible and $[M]^{-}$is a Type $I I_{1}$ $W^{*}$-algebra, by [28, Theorems 6.4 and 6.6] and [2, Theorem 8], respectively. Moreover, $M$ has a (unique) faithful normal centre-valued trace, $T$ (see [31, Sect. 18]). Since the centre, $Z(M)$, of $M$ is contained in the centre of $[M]^{-}$, and the closed convex hull of the set $\left\{U_{s_{i}} \ldots U_{s_{n}}(x): s_{i}\right.$ symmetries in $\left.M, n \in \mathbb{N}\right\}$ intersects $Z(M)$ nontrivially, [31, Proposition 1, Theorem 25], it follows from the properties of $T$ and the construction in [23,2.4.6], for example, that $T$ is the restriction of the faithful normal centre-valued trace on $[M]^{-}$, which we will also denote by $T$.

Lemma 5.1. Let $M$ be a Type $I I_{1} J W$-algebra and let e, $f$ be projections in $M$. Then

(i) $T(e)<T(f)$ (respectively $T(e)=T(f)$ ) if and only if $U_{s}(e)<f$ (respectively $\left.U_{s}(s)=f\right)$ for some symmetry $s$ in $M$.

(ii) If $e$ and $f$ are (von Neumann) equivalent in $[M]^{-}$, then $U_{s}(e)=f$, for some symmetry $s$ in $M$.

Proof. (i) Suppose that $T(e) \leqq T(f)$. By [31, Theorem 10] on Lemma 3.4(i), there is a symmetry $s$ and a central projection $z$ in $M$ such that $U_{s}(e \circ z) \leqq f \circ z$, 
$U_{s}(f \circ(1-z)) \leqq e \circ(1-z)$. Therefore,

$$
T\left(\left(\left(f-U_{s}(e)\right) \circ(1-z)\right)=T\left(U_{s}(f \circ(1-z))-e \circ(1-z)\right)=0 .\right.
$$

Hence $f-U_{s}(e)=\left(f-U_{s}(e)\right) \circ z \geqq 0$.

The rest is clear.

(ii) This follows from the above remarks together with (i) and [30, Chap. V, Corollary 2.8].

Lemma 5.2. Let $M$ be a Type $I I_{1} J W$-algebra and let $\phi$ be a probability measure on $M$. Suppose that $f, g$ are projections in $M$ with $\|f-g\|<\varepsilon<\frac{1}{2}$. Then $\mid \phi(f-\phi(g) \mid$ $<4 \sqrt{2} \varepsilon^{1 / 2}$.

Proof. We may suppose that $f \wedge g=0$. Let $s$ be a symmetry in $M$ such that $U_{s}(f)=g$ [see Remarks 3.4(ii)]. By [31, Theorem 17], there exist, in $M$, equivalent and orthogonal projections $f_{1}, f_{2}$ such that $f=f_{1}+f_{2}$. Put $g_{i}=U_{s}\left(f_{i}\right)$, for $i=1,2$. Then $\left\|f_{i}-g_{i}\right\| \leqq \sqrt{2}\|f-g\|^{1 / 2}<1, i=1,2$, by the proof of Corollary 3.7. By the above remarks, $T(f \vee g)=2 T(f)=4 T\left(f_{1}\right)$ and $T\left(f_{1} \vee g_{1}\right)=2 T\left(f_{1}\right)$. Hence, $T\left(\left(1-f_{1}\right) \wedge\left(1-g_{1}\right)\right)=T\left(1-f_{1} \vee g_{1}\right) \geqq 2 T\left(f_{1}\right)$. Thus, by Lemma 5.1 there exist, in $M$, orthogonal projections $p_{1}, q_{1}$, such that $p_{1} \sim q_{1} \sim f_{1} \sim g_{1}$ and $P_{1} \circ f_{1}=g_{1} \circ f_{1}$ $=p_{1} \circ g_{1}=0$; moreover, we can choose symmetries $s_{1}, t_{1}$ in $M$ such that $U_{s_{1}}\left(f_{1}\right)$ $=q_{1}=U_{t_{1}}\left(g_{1}\right)$. By Lemma 3.6, there exists a projection $h_{1} \in W\left[f_{1}, g_{1}, p_{1}\right]$ isoclinic to both $f_{1}, g_{1}$ with angle $\alpha=\frac{1}{2} \sin ^{-1}\left\|\left(f_{1}\left(1-g_{1}\right) f\right)^{1 / 2}\right\|$. Since, using Lemma 2.1, $W\left[f_{1}, h_{1}, q_{1} \circ s_{1}\right] \cong W\left[g_{1}, h_{1}, q_{1} \circ t_{1}\right] \cong M_{3}(\mathbb{R})_{s a}$ it follows that $\left|\phi\left(f_{1}\right)-\phi\left(g_{1}\right)\right|$ $\leqq 2\left\|f_{1}-g_{1}\right\|$, as in [7, Proposition 2.3] on using Gleason's theorem. Similarly, $\left|\phi\left(f_{2}\right)-\phi\left(g_{2}\right)\right| \leqq 2\left\|f_{2}-g_{2}\right\|$, and the proof is complete.

Given any probability measure, $\phi$, on the Type $I I_{1} \mathrm{JW}$-algebra $M$, in view of Lemma 5.2, the reversibility of $M$ and [31, Theorem 17], one can easily adapt [33, Proposition 2] to show that, via Theorem 3.8,

$$
\phi(f)=\phi(g f g)+\phi((1-g) f(1-g))+\phi(g f(1-g)+(1-g) f g)
$$

for any pair of projections $f, g$ in $M$.

In order to profit from the ingenious insights of Yeadon, [33, 34], we notice that $\phi$ extends to a state, $\bar{\phi}$, say, on the centre of $[M]^{-}$and define a finite trace, $\psi$, on $[M]^{-}$by $\psi(a)=\bar{\phi} T(a)$ for every $a$ in $[M]^{-}$. By restriction, $\psi$ is a finite trace on $M$, of course.

Armed with this construction and the above facts, examination of $[33,34]$ shows that Yeadon's methods can be carried over to the present context with very minor modifications, allowing us to state:

Theorem 5.3. Every measure on a Type $I I_{1} J W$-algebra extends to a positive linear functional on the whole algebra.

A JBW-algebra $M$ is said to be bounded Type $I_{\text {fin }}$ if $M=M_{1} \oplus M_{2} \oplus \ldots \oplus M_{r}$, where, for each $i, M_{i}$ is of Type $I_{n_{i}}$ with $n_{i}$ a finite integer.

Thus, in conclusion, on combining Theorems 3.8, 4.6, and 5.3, we have: 
Theorem 5.4. If $M$ is a JBW-algebra with bounded Type $I_{\text {fin }}$ part and without Type $I_{2}$ part then every measure on $M$ extends to a positive linear functional on $M$.

Corollary 5.5. If $M$ is a JBW-algebra without Type $I_{2}$ part then every countably additive measure on $M$ extends to a positive linear functional on $M$.

\section{References}

1. Aarnes, J.F.: Quasi-states on $C^{*}$-algebras. Trans. Am. Math. Soc. 149, 601-625 (1970)

2. Ajupov, S.A.: Extension of traces and type criterions for Jordan algebras of self-adjoint operators. Math. Z. 181, 253-268 (1982)

3. Alfsen, E.M., Shultz, F.W.: State spaces of Jordan algebras. Acta. Math. 140, 155-190 (1978)

4. Alfsen, E.M., Shultz, F.W., Hanché-Olsen, H.: State spaces of $C^{*}$-algebras. Acta. Math. 144, 267-305 (1980)

5. Alfsen, E.M., Shultz, F.W., St $\varnothing$ rmer, E.: A Gelfand Neumark theorem for Jordan algebras. Adv. Math. 28, 11-56 (1978)

6. Bunce, L.J.: Type I JB-algebras. Quart. J. Math. Oxford (2) 34, 7-19 (1983)

7. Christensen, E.: Measures on projections and physical states. Commun. Math. Phys. 86, 529-538 (1982)

8. Edwards, C.M.: On the facial structure of a JB-algebra. J. Lond. Math. Soc. 19, 335-344 (1979)

9. Edwards, C.M.: On the centres of hereditary JBW-subalgebras of a JBW-algebra. Math. Proc. Cambs. Phil. Soc. 85, 317-324 (1979)

10. Effros, E., Størmer, E.: Jordan algebras of self-adjoint operators. Trans. Am. Math. Soc. 127, 312-315 (1967)

11. Gil de Lamadrid, J.: Measures and tensors. Trans. Am. Math. Soc. 114, 98-121 (1965)

12. Gleason, A.M.: Measures on closed subspaces of a Hilbert space. J. Math. Mech. 6, 885-893 (1957)

13. Grothendieck, A.: Produit tensoriels topologiques et espaces nucléaires. Mem. Am. Math. Soc. 16 (1955)

14. Gunson, J.: Physical states on quantum logics. I. Ann. Inst. Henri Poincaré. Sect. A, XVII, 295-311 (1972)

15. Jacobson, N.: Structure and representation of Jordan algebras. Am. Math. Soc. Colloq. Publ. 39. Providence, RI: Am. Math. Soc. 1968

16. Janssen, G.: Die Struktur endlicher schwach abgeschlossener Jordan algebras. II. Manuscripta Math. 16, 307-332 (1975)

17. Jordan, P., von Neumann, J., Wigner, E.: On an algebraic generalisation of the quantum mechanical formalism. Ann. Math. 35, 29-64 (1935)

18. Mackey, G.W.: Quantum mechanics and Hilbert space. Am. Math. Monthly 64, 45-57 (1957)

19. Mackey, G.W.: The mathematical foundations of quantum mechanics. New York: Benjamin 1963

20. Matveichuk, M.S.: Description of the finite measures in semifinite algebras. Funct. Anal. Appl. 15, 187-197 (1982)

21. Pedersen, G.K.: $C^{*}$-algebras and their automorphism groups. New York: Academic Press 1979

22. von Neumann, J.: On an algebraic generalisation of the quantum mechanical formalism. Math. Sb. 1, 415-487 (1936)

23. Sakai, S.: $C^{*}$-algebras and $W^{*}$-algebras. Berlin, Heidelberg, New York: Springer 1971

24. Semadeni, Z.: Barach spaces of continuous functions, Vol. I. Monog. Matematyczne, 55. Warsaw: Polish Scientific Publ. 1971

25. Shultz, F.W.: On normed Jordan algebras which are Banach dual spaces. J. Funct. Anal. 31, 360-376 (1979)

26. Stacey, P.J.: Type $I_{2}$ JBW-algebras. Quart. J. Math. Oxford (2), 33 115-127 (1982) 
27. St $\varnothing$ rmer, E.: On the Jordan structure of $C^{*}$-algebras. Trans. Am. Math. Soc. 120, 438-447 (1965)

28. St $\varnothing$ rmer, E.: Jordan algebras of Type $I$. Acta Math. 115, 165-184 (1965)

29. St $\varnothing \mathrm{rmer}$, E.: Irreducible Jordan algebras of self-adjoint operators. Trans. Am. Math. Soc. 130, 153-166 (1968)

30. Takesaki, M.: Theory of operator algebras. I. Berlin, Heidelberg, New York: Springer 1979

31. Topping, D.: Jordan algebras of self-adjoint operators. Mem. Am. Math. Soc. 53 (1965)

32. Wright, J.D.M.: Jordan $C^{*}$-algebras. Mich. Math. J. 24, 291-302 (1977)

33. Yeadon, F.J.: Measures on projections in $W^{*}$-algebras of Type II. Bull. Lond. Math. Soc. 15, 139-145 (1983)

34. Yeadon, F.J.: Finitely additive measures on projections in finite $W^{*}$-algebras (to appear)

Communicated by H. Araki

Received November 28, 1983; in revised form September 8, 1984 